\title{
WP 15-17
}

\author{
Haluk Yener
}

Department of Business Administration, Istanbul Bilgi University, Turkey

\author{
Thanasis Stengos
}

Department of Economics, University of Guelph, Canada

The Rimini Centre for Economic Analysis, Italy

\author{
M. Ege Yazgan
}

Department of Economics, Kadir Has University, Turkey

The Rimini Centre for Economic Analysis, Italy

\section{SURVIVAL MAXIMIZING LEVERAGE OF AN ECONOMY: The CaSe OF GReECE}

Copyright belongs to the author. Small sections of the text, not exceeding three paragraphs, can be used provided proper acknowledgement is given.

The Rimini Centre for Economic Analysis (RCEA) was established in March 2007. RCEA is a private, nonprofit organization dedicated to independent research in Applied and Theoretical Economics and related fields. RCEA organizes seminars and workshops, sponsors a general interest journal The Review of Economic Analysis, and organizes a biennial conference: The Rimini Conference in Economics and Finance (RCEF) . The RCEA has a Canadian branch: The Rimini Centre for Economic Analysis in Canada (RCEACanada). Scientific work contributed by the RCEA Scholars is published in the RCEA Working Papers and Professional Report series.

The views expressed in this paper are those of the authors. No responsibility for them should be attributed to the Rimini Centre for Economic Analysis. 


\title{
Survival Maximizing Leverage of an Economy: The Case of Greece
}

\author{
Haluk Yener ${ }^{1} \quad$ Thanasis Stengos $^{2} \quad$ M. Ege Yazgan ${ }^{3}$
}

\begin{abstract}
In this paper, we solve two problems related to growth and survival probability maximization of an economy. We assume that the problems are subject to the stochastic net worth model introduced by Fleming and Stein (2004) and apply the techniques of stochastic optimal control theory in order to find our results. Via the results, we first establish the analytical specifications for the minimum goal reaching and maximum survival times of an economy. Given these, we show that the growth maximizing strategy enhances both the survival and goal reaching times besides its growth enhancing property. We also provide a closed form solution for the estimated debt crisis time of a non-resilient economy. Furthermore, by considering a fixed proportional consumption rate, we specify survival probability maximizing leverage as a function of this rate and show how optimal leverage strategy changes as the conditions of the economy changes. Mainly, we show that under bad economic conditions, bold leverage strategy must be followed at the expense of growth for the survival probability maximization. On the other hand, under good economic conditions, timid strategies are better as excessive risk taking must be avoided for the survival probability maximization. Again, for the same objective, we show that as an economy becomes less self sufficient, then, it needs to resort to external funds in order to finance its consumption, which may in turn lead excessive borrowing that may end up with debt crisis as it did in Greece.
\end{abstract}

JEL Classification: C61, D81, F34, F43

Keywords: Foreign debt, sustainable growth, debt crises, growth maximization, international finance, current account deficit.

\footnotetext{
${ }^{1}$ Department of Business Administration, Istanbul Bilgi University, Santral Campus, 34060, Eyup, Istanbul, TURKEY; haluk.yener@bilgi.edu.tr

${ }^{2}$ Department of Economics, University of Guelph, Guelph, Ontario, N1G 2W1, CANADA; tstengos@uoguelph.ca

${ }^{3}$ Department of Economics, Kadir Has University, 34083, Cibali, Istanbul, TURKEY; ege.yazgan@khas.edu.tr
} 


\section{Introduction}

The recent debt crisis in Greece that was precipitated in 2009 has been the focus of a heated discussion in policy circles in Europe and around the world regarding the sustainability of a country's debt (taken mainly to denote public debt) and its ability to meet its future obligations. If a debt trajectory is deemed to be unsustainable that would have severe repercussions for future growth and stability for the country in question, especially in the presence of negative demographic projections that would further hinder fiscal spending in the future, (see Cecchetti et al. (2011a)). The main negative link of high debt to economic growth besides meeting the repayment obligations rests on the potential crowding out effect of higher public debt on private investment (see Mankiw and Elmendorf (1999)). However, an additional important factor is that investors may interpret the high debt-to-GDP ratios as the result of time inconsistent policies that have led to the crisis, something that now has forced a country like Greece to impose severe austerity measures on their citizens and adopt fiscal discipline in order to restore their credibility.

In the empirical literature, following the highly influential study by Reinhart and Rogoff (2010), there is a general consensus that there exists a weak relationship between government (public) debt and economic growth for countries with debt below $90 \%$ of the GDP, yet the effect is strongly negative for countries with a debt-to-GDP ratio over $90 \%$. There has been many more recent studies that identify the non-linear threshold effects of the (public) debt and growth nexus by using the threshold regression methods, see Cecchetti et al. (2011b), Caner et al. (2010) and Kourtellos et al. (2013) to mention a few. All these studies only consider public debt and not the overall debt, both public and private, an economy faces and there is little distinction between internal and external debt holdings.

A recent paper that has tried to address the above point is Yener, Stengos \& Yazgan (2014). It used a different framework that allows for the total overall external debt to be taken into account and examined the seeds of the Eurozone debt crisis. The paper follows Fleming and Stein (2004) to offer an early warning signal of the debt crisis that amounts to the absence of an admissible leverage strategy for turning the economy around during a downturn if the fundamentals are not sufficiently favorable. In that context, one can examine precisely how excessive risk taking due to over-borrowing is a danger to an economy.

In the current paper, we first extend Yener, Stengos \& Yazgan (2014) to predict a possible debt crisis of an economy by applying the study ofBrowne (1999) ${ }^{4}$ to our case and

\footnotetext{
${ }^{4}$ In his study, Browne considers an investor who dynamically trades a risky portfolio, consisting of a risk-free and $N$ risky assets. The aim of the investor is to beat an exogenously given stochastic benchmark by maximizing (minimizing) the expected time to stay above (reach) a certain fractional value of the benchmark, maximizing the probability of reaching a higher fractional value of the benchmark
} 
borrowing from his analysis. The extension involves solving the survival time problem ${ }^{5}$ for an economy that is deemed to be non-resilient under the conditions provided by Yener, Stengos \& Yazgan (2014). In this way, a closed form solution is provided for the expected time of a possible debt crisis in an economy. Furthermore, by using the optimal results, we also show that the growth maximizing leverage strategy does improve the goal reaching and survival times of an economy. In addition to the prediction model, we also solve for the optimal leverage strategy that will maximize the survival probability of an economy.

Via our solution to the second problem, we first show the relation between the fundamentals of an economy and a risk aversion type parameter that appears in the survival probability maximizing leverage strategy. Through this, we observe how the leverage strategy changes as the conditions in an economy change and see why a survival probability maximizing economy would pursue leverage strategies whose risks are in excess of that of the growth maximizing leverage strategy. Mainly, via our analysis, we devise that under bad economic conditions excessive risk must be taken at the expense of growth, while excessive risk must be avoided under good economic conditions. Furthermore, we also establish the effect of the consumption on the survival probability maximizing leverage strategy and show the conditions under which exposure to external funds is better than no exposure at all for an economy. Here, we found that as an economy becomes less self-sufficient, then it must resort to external resources in order to meet the excess consumption, hence to maximize its survival probability.

The starting point in the approach that we use is the model of Fleming and Stein (2004) that consists of a stochastic net worth process given as the difference between the capital and debt stock in an economy. Their aim is to derive the optimal leverage strategy of an economy by benefiting from the pioneering approach of Merton (see Merton (1971) and Merton (1990)) who solves the optimal investment strategy of an investor dynamically trading a stochastic portfolio in frictionless markets. The application of the approach is possible thanks to the similarity of the net worth model to a stochastic portfolio process. However, the main difference lies in the sources of risks which are mainly due to the uncertainty in the return on capital and the effective rate of return on net foreign assets. Furthermore, the risk arising from the net foreign assets will effect the economy as long as it is open. In other words, leverage is used if the economy becomes open to the external world via debt exposure. Under the aforementioned form of the model, Fleming and Stein (2004) derives the optimal leverage strategy, then, they use it as a benchmark to detect a possible debt crisis; if the actual leverage rises above a

without first hitting its lower fractional value, and maximizing/minimizing the expected discounted reward/penalty. Browne then provides the analytical solutions of the optimal investment strategies of the investor by solving the three aforementioned problems via the application of the techniques of stochastic optimal control. Furthermore, he conducts the analyses of the results in order to connect three problems around the theme of market favorability so that the conditions of the markets are related to the investment strategies of the investor.

${ }^{5}$ We also provide the solution to the goal reaching problem for the sake of completeness in our argument. 
significant level from the benchmark, it then signals a possible debt crisis as such level is deemed to be unsustainable.

A related study that looks at the same model is an earlier paper by Fleming and Stein (2001). In that study, they considered discrete time setting and assumed an uncertain productivity rate. Given this setup, they construct the model under no default constraint and provide a maximal difference level between the actual and the optimal debt levels in order to use the difference as an early warning signal of a possible debt crisis. That is, the deviation of the actual leverage from a level that could be designated by the fundamentals signals debt crisis. This claim is made more robust under the empirical assessment of Stein and Paladino (2001). The study shows how the model can be used as an early warning signal for possible debt crises via the analysis of the results of a default group of twenty-one countries that defaulted on the debt during the period 1978-99 and a group of thirteen countries as the control group.

The model has also been used as an early warning signal of various crises that happened around the world ${ }^{6}$; see for example Stein (2005) on the Asian crisis that happened during 1997-1998 and on the Argentinian crisis in 2001, Stein (2007) on the analysis of the US current account deficit, and Stein (2010) on US Agricultural debt crisis during 1980s and the subprime mortgage crisis in 2007. As a more relevant study to our case, Stein (2011) examined the underlying factors of the European debt crisis. Mainly, the study shows that while the private sector borrowing, particularly housing, was the main reason behind the crises in Ireland and Spain, the cyclically adjusted structural deficits in Greece and Portugal were the main reason of the crises in these countries.

Within the context of Stein (2011), Yener, Stengos \& Yazgan (2014) analysed the seeds of the European debt crisis by using national account data. This is different from Stein (2011) that interpreted and used various data from secondary sources related to interest rates, gross domestic product, government structural balance, government net debt, residential property prices, rent prices, and return on assets along with the optimal leverage strategy specification in order to reach the conclusions stated in the paper. Furthermore, Yener, Stengos \& Yazgan (2014) also show how excessive risk taking along with deteriorating fundamentals led to the crisis. Mainly, the study establishes the growth enhancing property of the growth maximizing leverage strategy and uses it as a limiting case for the detection of a possible debt crisis. That is, if the actual leverage strategy is higher than the growth maximizing one, then the deviation might indicate a problem in future debt payments, as pursuing multiples of growth maximizing leverage leads to lower growth with excessive risk in the evolution of the net worth process of an economy.

However, excessive leverage may not solely be enough to indicate a debt crisis because the fundamentals of an economy may save it from a possible default. That is, if the fundamentals of an economy are strong, then, that economy may move into new equilibrium without defaulting on its debt in case of a downturn. Therefore, there is a need for a

\footnotetext{
${ }^{6}$ Two books, Stein (2006) and Stein (2012), contain the collection of some notable studies that applied the model.
} 
measure of the strength of the fundamentals in an economy when interpreting the level of the actual leverage relative to an optimal one. To realize this, Yener, Stengos \& Yazgan (2014) applies the argument in Browne (1999), and shows that there is no admissible leverage strategy for turning the economy around in an economic downturn when the fundamentals are not favourable. Hence, excessive risk taking by an economy when its fundamentals are not strong enough leads to debt problems.

In the current paper, we reiterate the aforementioned argument more thoroughly and extend it to a prediction study by providing a closed form solution for the estimation of a crisis time. Our application to the case in Greece shows that the model predicts the estimated crisis time in around mid-2008 with an upper boundary indicating the beginning of September 2009. In the second part, we apply the theoretical findings of the survival probability maximization problem to have a further analysis of the Greek debt crisis. Our results, show that the realized leverage strategy of Greece did indeed increase after 2004 more steeply, indicating the use of foreign funds for non-productive resources. Furthermore, the association of expansionary policy with deteriorating self-sufficiency was a sign of the recent debt crisis in Greece; hence excessive risk taking at the expense of growth.

To find the results, we first construct the stochastic net worth process model in subsection 2.1 of section 2. Then, we proceed to solve the first problem related to finding the optimal leverage strategy that maximizes or minimizes the expected time to fulfil a certain aim in subsection 2.2. There, we also provide the expected survival time value until a possible debt crisis. Next, we solve the problem for maximizing the survival probability in subsection 2.3. The analysis of the results are given in each section. The application of the results are however realized in section 3 (see subsections 3.1 and 3.2). As mentioned previously, we use Greece as a case for the application of the theoretical findings in the paper. In this way, we also have a better understanding of our findings and observation of the underlying reasons behind a major crisis that happened recently in Europe. The proofs, on the other hand, are given in the appendices. In the proofs, for the verification of the optimality, we modify the approach presented in Björk (2004) (see also Fleming and Soner (1993)) to our case and used localization argument. We conclude by summarizing the findings in section 4 .

\section{The Framework}

\subsection{The Model}

In this section, we first construct the underlying mathematical model for our analysis. To this end, we consider infinite horizon for an economy that is modeled under a complete filtered probability space $\left(\Omega, \mathcal{F},\left\{\mathcal{F}_{t}\right\}_{0 \leq t<\infty}, \mathbb{P}\right)$. Here, $\mathbb{P}$ denotes the real measure, and we let $\left\{\mathcal{F}_{t}\right\}_{0 \leq t<\infty}$ be the $\mathbb{P}$-augmentation of the natural filtration $\mathcal{F}_{t}^{W}:=\sigma\{W(u) \mid u \leq t\}$, 
where $W(\cdot)$ is the 2-dimensional Brownian motion $W(t):=\left(W_{1}(t), W_{2}(t)\right)^{\prime}, 0 \leq t<\infty$ defined on our complete probability space. In the probability model, $\Omega$ is the set of all possible events in an economy and the sigma algebra $\mathcal{F}_{t}$, which is the set of all subsets of $\Omega$, provides information about the status of the economy. Furthermore, the filtration $\left\{\mathcal{F}_{t}\right\}_{0 \leq t<\infty}$ represents the evolution of the uncertainties in the same economy.

The uncertainties in the economy are in turn modeled by two correlated Brownian motions $W_{1}(\cdot), W_{2}(\cdot)$. That is, we have

$$
d W_{1}(t) d W_{2}(t)=\rho d t
$$

where $|\rho| \leq 1$ denotes the constant correlation coefficient. We consider two sources for the uncertainties, which are namely the effective rate of return on debt and the return on capital. We specify these processes respectively as

$$
\begin{aligned}
r(t) d t & =r d t+\sigma_{1} d W_{1}(t) \\
b(t) d t & =b d t+\sigma_{2} d W_{2}(t)
\end{aligned}
$$

where $b$ is the mean rate of change in the return on capital and $r$ is the mean effective rate of return on loans. Note that, we could also consider deterministic mean rates, however, to simplify the notation, we let them be constant without loss of generality. Furthermore, we also assume that the standard deviations of our processes, $\sigma_{1}>0, \sigma_{2}>0$, are constants.

To construct the model we first let $N(t)$ denote the quantity of units of capital at time $t, P(t)^{7}$ be the unit price of capital at time $t$, and $K(t)>0$ be the capital stock at time $t$. Then, $K(t)$ is given by

$$
K(t)=N(t) P(t)
$$

Moreover, provided that $I(t)$ is the investment rate, the change in the capital stock is, from (2.4), equal to

$$
d K(t)=N(t) d P(t)+P(t) d N(t)=K(t) \frac{d P(t)}{P(t)}+I(t) d t
$$

where $\left\{d P_{t} / P_{t}, t \geq 0\right\}$ denotes the stochastic capital gain. We observe from (2.5) that capital stock is in increasing relationship with the capital gain and the investment rate. Given, the capital stock identity, we then proceed to define the gross domestic product, $Y(t)$, of an economy with the proportional relationship

$$
Y(t) d t=\alpha(t) K(t) d t
$$

where $\{\alpha(t), t \geq 0\}$ is the stochastic productivity rate and denotes the proportionality of the gross domestic product to the capital stock. That is, the added value in an economy is the product of the productivity rate and the capital stock.

\footnotetext{
${ }^{7}$ As Stein (2007) explains, $P(t)$ is a measure of quality of the capital.
} 
Next, we let $L(t)$ denote the debt value of an economy at time $t$, and write the balance equation for the change in debt value as

$$
d L(t)=[r(t) L(t)+I(t)+C(t)-Y(t)] d t
$$

where $C(t)>0$ is the consumption level at time $t$. As we observe from the above equation, the change in debt is the result of the difference between the sum of the net transfer payments $r(t) L(t)$, investment rate $I(t)$, the consumptions rate $C(t)$ and the gross domestic product $Y(t)$. In this regard, as Fleming and Stein (2004) explained, the balance equation (2.7) is equal to the current account deficit, and $L(t)$ therefore denotes the value of the net foreign assets of an economy. Furthermore, we see that the balance equation for debt is in increasing relationship with investment and consumption rates and in decreasing relationship with the added value. Mainly, if $I(t)+C(t)-Y(t)$ denotes the trade balance, then an economy will become debtor, $L(t)>0$, to the rest of the world, the lower its value addition relative to its rate of investment and consumption, and creditor, $L(t)<0$, the higher its value addition is relative to its rate of investment and consumption.

On the other hand, we let $X(t)$ be the net worth process given by

$$
X(t)=K(t)-L(t)
$$

and specify the consumption proportionally by $C(t, X(t))=c X(t)$, where $c \geq 0^{8}$ is exogenously given constant rate of consumption. We consider a constraint of the form $X(t)>0$ so that debt is not financed by further borrowing. In this way, a Ponzi scheme is avoided.

Next, by substituting the equations (2.2) and (2.5)-(2.7) into the equation (2.8), the change in the net worth becomes equal to

$$
\begin{aligned}
d X(t) & =d K(t)-d L(t) \\
& =\left[\alpha(t)+\frac{d P(t)}{P(t)}\right] K(t)-C(t) d t-r L(t) d t-\sigma_{1} L(t) d W_{2}(t)
\end{aligned}
$$

We see from equation (2.9) that the net worth of an economy is increasing with increasing productivity and capital gain. In other words, the model captures the benefit of the technical progress and capital gain as they lead to an increase in the capital stock and value addition of an economy. However, for a given level of technical progress and capital gain, we observe from equation (2.5) that an increase in the capital stock may also be due to increasing investment. While additional investment improves the capital stock, from (2.7), it also leads to an increase in the debt level. Therefore, the effect of a change in investment to capital stock is offset by the effect of a change in investment to debt

\footnotetext{
${ }^{8}$ Here as well, we could consider deterministic proportional consumption rate. Nevertheless, we let it be constant without loss of generality.
} 
stock, and as a result $I(t)$ is not present in equation (2.9). On the other hand, increasing consumption and increasing debt as evident from equations (2.7) and (2.8), lead to a decline in the net worth. Especially, we may see from (2.7) that when consumption increases, debt increases, which in turn lowers the value of the net worth for a given level of capital. Therefore, any foreign borrowing directed towards consumption causes a decline in the net worth of an economy, which shows the damage of spending resources in a non-productive manner.

In order to model the stochastic part $\{\alpha(t)+d P(t) / P(t), t \geq 0\}$, we use equation (2.3) provided for modeling the stochastic rate of return on capital. Then, by substituting this equation into (2.8), we write (2.9) as

$$
d X(t)=[b X(t)+(b-r) L(t)-C(t)] d t-L(t) \sigma_{1} d W_{1}(t)+(X(t)+L(t)) \sigma_{2} d W_{2}(t) .
$$

Our goal is to express the above equation via a control process $\boldsymbol{f}(t)=L(t) / X(t)$ named as the leverage strategy. Given the rate of consumption, the mean return on capital, the mean effective rate of return on the net foreign assets, and the risks arising from the last two aforementioned variables, an economy seeks an appropriate level of leverage to control the evolution of its net worth. Such control however must be realized within the admissible limits; we say that the leverage strategy is admissible for an initial net worth amount $x$, denoted by $\boldsymbol{f} \in \mathcal{A}(x)$, if $\boldsymbol{f}(t)$ is $\left\{\mathcal{F}_{t}\right\}$-progressively measurable and satisfies $\int_{0}^{t} f^{2}(s) d s<\infty$ for $t<\infty$. In words, leverage level must depend on the observed value of the net worth process and its value must be finite since resources are scarce. Then, the net worth process associated to an admissible leverage process $\boldsymbol{f}(t)$ is the solution of the stochastic differential equation

$$
\begin{aligned}
d X^{f}(t)= & {\left[\left(b_{t}-c_{t}\right)+\left(b_{t}-r_{t}\right) \boldsymbol{f}(t)\right] X^{f}(t) d t-\boldsymbol{f}(t) X^{f}(t) \sigma_{1} d W_{1}(t) } \\
& +(1+\boldsymbol{f}(t)) X^{f}(t) \sigma_{2} d W_{2}(t) \\
X(0)= & x .
\end{aligned}
$$

Under an admissible strategy, the closed form solution to the above equation is

$$
\begin{aligned}
X^{f}(t)= & x \exp \left\{\int _ { 0 } ^ { t } \left[(b-c)+\boldsymbol{f}(s)(b-r)-\frac{1}{2}\left(\boldsymbol{f}^{2}(s) \sigma_{1}^{2}+(1+\boldsymbol{f}(s))^{2} \sigma_{2}^{2}\right)\right.\right. \\
& \left.\left.-\boldsymbol{f}(s)(1+\boldsymbol{f}(s)) \rho \sigma_{1} \sigma_{2}\right] d s-\int_{0}^{t} \boldsymbol{f}(s) \sigma_{1} d W_{1}(s)+\int_{0}^{t}(1+\boldsymbol{f}(s)) \sigma_{2} d W_{2}(s)\right\} .
\end{aligned}
$$

From the above specification, we have $X^{f}(t)>0$ for $t<\infty$. In this way, Ponzi scheme is avoided. Furthermore, we assume that there are no frictions, implying that the leverage strategy can be changed instantaneously and costlessly. Note that changing the leverage strategy is equivalent to changing the capital strategy, therefore controlling the rate of 
investment, since from equation (2.8) the fractional capital amount at time $t$ is given by $\boldsymbol{k}(t)=K(t) / X(t)=1+\boldsymbol{f}(t)$. However, we must always have $\boldsymbol{f}(t)>-1 \forall t$ as $K(t)>0$. Otherwise, we would have $K(t) \leq 0$, implying the entire use or short selling opportunity of a capital for an economy. Such case is restricted as an economy cannot use all of its capital or borrow the capital of another economy to benefit further from lending. Therefore, there must a be certain level of positive capital left for use so that the economy stays afloat. As a result, we select a level $\theta \in(-1,0)$ and if the mathematical solution of the problems we are solving leads the optimal leverage strategy at time $t$, denoted by $\boldsymbol{f}^{*}(t)$, to be $\boldsymbol{f}^{*}(t) \leq \theta$, then we set $\boldsymbol{f}^{*}(t)=\theta$.

\subsection{Growth Maximization}

If we set $\boldsymbol{f}(t)=0$ in equation (2.12), we obtain the net worth process of a closed economy. That is, we obtain

$$
X(t)=x \exp \left\{\Lambda t+\sigma_{2}^{2} W_{2}(t)\right\}, \quad \text { where } \Lambda=b-c-\frac{1}{2} \sigma_{2}^{2},
$$

implying that, for a given level of average rate of return on capital, the downside to the growth of the net worth process arises from an increase in the rate of consumption and/or in the risk level of the return on capital. Especially, if an economy is unable to meet its consumption from its own internal resources, it may then have to open to external funds. Opening to external world, however, brings extra risk arising from the uncertainty in the effective rate of return on debt. Therefore, an economy needs to select its leverage to enhance growth given the underlying risks. To enhance growth, an economy may pursue growth maximizing leverage strategy (see Yener, Stengos \& Yazgan (2014)), which is given by

$$
\boldsymbol{f}^{*}= \begin{cases}\frac{b-r}{\sigma^{2}}+\alpha(\rho \kappa-1), & \text { if } \theta<\frac{b-r}{\sigma^{2}}+\alpha(\rho \kappa-1) \\ \theta, & \text { if } \frac{b-r}{\sigma^{2}}+\alpha(\rho \kappa-1) \leq \theta\end{cases}
$$

where $\sigma^{2}=\sigma_{1}^{2}+\sigma_{2}^{2}-2 \rho \sigma_{1} \sigma_{2}, \alpha=\sigma_{2}^{2} / \sigma^{2}$, and $\kappa=\sigma_{1} / \sigma_{2}$. Then, substituting (2.14) in equation (2.12) yields for $t<\infty$

$$
X^{*}(t)=\left\{\begin{array}{cl}
x \exp \left\{\beta t-\left(\frac{b-r}{\sigma^{2}}+\alpha(\rho \kappa-1)\right) \sigma_{1} W_{1}(t)\right. & \\
\left.+\left(1+\frac{b-r}{\sigma^{2}}+\alpha(\rho \kappa-1)\right) \sigma_{2} W_{2}(t)\right\}, & \text { if } \theta<\frac{b-r}{\sigma^{2}}+\alpha(\rho \kappa-1) \\
x \exp \left\{\beta t-\theta \sigma_{1} W_{1}(t)+\theta \sigma_{2} W_{2}(t)\right\}, & \text { if } \frac{b-r}{\sigma^{2}}+\alpha(\rho \kappa-1) \leq \theta,
\end{array}\right.
$$

where $\beta$, the trend of the growth maximized net worth process, is given by

$$
\beta= \begin{cases}\Lambda+\frac{1}{2}\left[\frac{b-r}{\sigma}+\sigma(\alpha(\rho \kappa-1))\right]^{2}, & \text { if } \theta<\frac{b-r}{\sigma^{2}}+\alpha(\rho \kappa-1) \\ \Lambda+\sigma^{2} \theta\left[\frac{b-r}{\sigma^{2}}+\alpha(\rho \kappa-1)-\frac{1}{2} \theta\right], & \text { if } \frac{b-r}{\sigma^{2}}+\alpha(\rho \kappa-1) \leq \theta\end{cases}
$$


We observe that the growth maximizing leverage strategy is not dependent on the proportional rate of consumption. Furthermore, as explained in Yener, Stengos \& Yazgan (2014), we see that pursuing this strategy when opening to external funds adds to the growth rate of an economy while bringing extra uncertainty arising from the net effective rate of return on net foreign assets. Given exposure to foreign funds, then, considering the first case (i.e. $\theta<\boldsymbol{f}^{*}$ ), a growth maximizing economy becomes debtor whenever $r<b+\rho \sigma_{1} \sigma_{2}-1$ and a creditor if $r>b+\rho \sigma_{1} \sigma_{2}-1$. On the other hand, if an economy is a debtor, we have $\partial \beta / \partial r<0$, and if creditor $\partial \beta / \partial r>0$. In words, increasing average effective rate of return on net foreign assets causes a decline (increase) in the growth rate of a debtor (creditor) economy. Growth rate also declines with increasing $c, \sigma_{1}, \sigma_{2}$, and increases with increasing $b$. That is, while increasing average rate of return on capital adds to the growth, even if it might lead, from equation (2.14), to further borrowing for a growth maximizing economy, increasing propensity to consume and risk levels impedes sustainable increase in the value of the net worth process.

If changes in the factors of an economy becomes detrimental in a way that $\beta<0$, we say by referring to Yener, Stengos \& Yazgan (2014) (which applies the arguments of Browne (1999)) that such economy becomes a survival time maximizing economy. More clearly, as long as a growth maximizing economy has negative growth rate due to high risk levels and/or propensity of consumption, then, it can use the growth maximizing leverage strategy only to prevent its net worth decline toward undesired level at the longest expected time possible. However, even if a strategy leads to the delay of hitting to the undesired level, it cannot save the trend of the net worth process from being negative. In this case, as time goes on, the net worth process approaches further to zero, implying higher increase in the level of debt stock relative to capital stock.

Provided that an economy does not follow growth maximizing strategy, the decline will be realized at a faster rate. As a result, the actual leverage strategy $\boldsymbol{f}$ increases and provides a signal for a possible debt crisis. Especially, if it exceeds the growth maximizing case, such leverage strategy may be deemed to be unsustainable as it causes very high risk with lower growth. Therefore, as shown in Yener, Stengos \& Yazgan (2014), if $\beta<0$ and $\boldsymbol{f}-\boldsymbol{f}^{k}>0$, then the resilience ${ }^{9}$ of an economy against a possible debt crisis may be questionable since the economy is in bad shape due to negative growth and the risk level is beyond that an economy would face with the growth maximizing leverage strategy ${ }^{10}$.

On the other hand, the case when $\beta>0$ and $\boldsymbol{f}-\boldsymbol{f}^{k}>0$, is used to denote that an over-leveraged economy may always find an admissible leverage strategy to save itself from a possible debt crisis as long as the factors of the economy are in good shape. In other words, if the factors of the economy lead to the growth of the net worth process, a leverage strategy that hinders an economy from deterioration will always exist. In this

\footnotetext{
${ }^{9}$ We refer the readers to Yener, Stengos \& Yazgan (2014) for further details on the non-resilience condition of an economy.

${ }^{10}$ That is, when the growth maximizing strategy is exceeded, an economy not only has lower growth, but also has much higher risk than the level it faces under growth maximizing strategy.
} 
case (that is when $\beta>0$ ), the growth maximizing strategy minimizes the expected goal reaching time of an economy.

Next, we formulate the problem to find the optimal survival and goal reaching times of the net worth process of an economy. Mainly, our goal is to show that an economy that is open to the rest of the world under the growth maximizing leverage strategy will be better off than remaining closed, when it aims to reach a certain target at the quickest possible time under good economic conditions, or to survive at the longest possible time without debt problems under bad economic conditions. To this end, we consider two barrier points $U$ and $L$ such that $L<x<U$, and let

$$
\tau_{U}^{f}=\inf \left\{t>0 \mid X^{f}(t) \geq U\right\}
$$

be the first time the net worth process crosses the higher net worth level under a leverage strategy $\boldsymbol{f}$, and

$$
\tau_{L}^{f}=\inf \left\{t>0 \mid X^{f}(t) \leq L\right\}
$$

be the first time the net worth process crosses the lower net worth level under a leverage strategy $f$. Given the definitions for the hitting times, we then proceed to define the objective to maximize the expected survival time as

$$
\underline{F}(x)=\sup _{f \in \mathcal{A}(x)} \mathbb{E}_{x}\left[\tau_{L}^{f}\right]
$$

and the objective to minimize the expected goal reaching time as

$$
\bar{F}(x)=\inf _{f \in \mathcal{A}(x)} \mathbb{E}_{x}\left[\tau_{U}^{f}\right]
$$

where $\mathbb{E}_{x}[\cdot]=\mathbb{E}[\cdot \mid X(0)=x]$. Then, we provide the solutions of the two objective functions in the following proposition.

Proposition 2.1. Let the net worth process $\left\{X^{f}(t), 0 \leq t<\infty\right\}$ be given by equation (2.12). Furthermore, if we have $\beta \in \mathbb{R} \backslash\{0\}$, a parameter that denotes the condition of the economy and is given by equation (2.16), then:

(i) When $\beta<0$ (bad economic condition), the optimal value function is given by

$$
\underline{F}(x)=\frac{1}{|\beta|} \ln \left(\frac{x}{L}\right) \quad \text { for } x \in[L, \infty) .
$$

(ii) Next, when $\beta>0$ (good economic condition), the optimal value function is given by

$$
\bar{F}(x)=\frac{1}{\beta} \ln \left(\frac{U}{x}\right) \quad \text { for } x \in(0, U] .
$$

The optimal leverage strategy for both problems is, in turn, the growth maximizing leverage strategy specified in equation (2.14). 
Proof. Please see Appendix A

Since the growth maximizing leverage strategy enhances growth, we have from equations (2.13) and (2.16), $\Lambda<\beta$. Furthermore, without leverage the expected survival time and the expected goal reaching time would respectively be equal to ${ }^{11}$

$$
\begin{aligned}
& \underline{F}(x)=\frac{1}{|\Lambda|} \ln \left(\frac{x}{L}\right), \text { where } \Lambda<0 \text { and } x \in[L, \infty) \\
& \bar{F}(x)=\frac{1}{\Lambda} \ln \left(\frac{U}{x}\right), \text { where } \Lambda>0 \text { and } x \in(0, U] .
\end{aligned}
$$

Therefore, for the survival time, because we have $\Lambda<\beta<0$, we write

$$
\frac{1}{|\Lambda|} \ln \left(\frac{x}{L}\right)<\frac{1}{|\beta|} \ln \left(\frac{x}{L}\right)
$$

and for the goal reaching time, because $0<\Lambda<\beta$, we have

$$
\frac{1}{\beta} \ln \left(\frac{U}{x}\right)<\frac{1}{\Lambda} \ln \left(\frac{U}{x}\right) .
$$

That is, by exposing itself to external funds via the growth maximizing leverage strategy, an economy not only enhances its growth rate but also enhances its expected survival or goal reaching times. Especially, for the survival maximizing economy, growth maximizing leverage strategy may be used as a benchmark that may signal the maximum expected time the net worth process will stay above a certain lower threshold level $L$ (a condition that may be used as a signal of a possible debt crisis). That is, the maximum time a non-resilient economy may avoid a debt problem is given by (2.19). Under any other leverage strategy, the time for a possible debt crisis must therefore be shorter than the maximum time denoted via the growth maximizing strategy. Furthermore, since the non-resilience condition implies $\boldsymbol{f}-\boldsymbol{f}^{*}>0$, where $\boldsymbol{f}$ is an actual leverage strategy in excess of the growth maximizing strategy, the excessive actual leverage strategy may in turn be represented as a multiple of the growth maximizing strategy. To this end we write from Fleming and Stein (2004) (see also Yener, Stengos \& Yazgan (2014))

$$
\mathbf{f}^{*}(x)=-\frac{1}{p} \frac{b-r}{\sigma^{2}}+\alpha(\rho \kappa-1)
$$

which is the optimal leverage strategy obtained by maximizing a power type utility function given by $U(x)=x^{1+p} / 1+p$, where $p \in(-\infty, 0) \backslash-1$ is the risk aversion parameter. Then, we observe that when $p \in(-1,0)$, the leverage strategy given in equation $(2.23)$ is a multiple of the growth maximizing strategy. In this case, we write the following corollary.

\footnotetext{
${ }^{11}$ The specifications in (2.19) and (2.20) may be obtained by setting the leverage strategy equal to zero in the proof of proposition 2.1 .
} 
Corollary 2.1. Consider a non-resilient economy. Then, the survival time under the leverage strategy given in equation (2.23) is equal to

$$
\underline{F}^{p}(x)=\frac{1}{\left|\beta^{p}\right|} \ln \left(\frac{x}{L}\right) \quad \text { for } x \in[L, \infty),
$$

where $\beta^{p} i s^{12}$

$$
\beta^{p}=\beta-\frac{1}{2}\left(1+\frac{1}{p}\right)^{2} \frac{(b-r)^{2}}{\sigma^{2}},
$$

Proof. The proof of the corollary follows straightforwardly from the proof of the main problem in this section; we set $\boldsymbol{f}$ in the Hamilton-Jacobi-Bellman equation equal to the power utility maximizing control policy, then solve for the function that sets the equation equal to zero. In this way, we find the unique solution as given in (2.24) to a Dirichlet-type problem.

By using equation (2.24) in the above corollary, we can then estimate the time the net worth process of an economy spotted as non-resilient will hit a lower threshold level $L$. Via proper selection of $L$, we may in turn see, if estimated hitting time given by the closed form formula in (2.24) may be used to predict possible debt problem in a non-resilient economy.

Remark 2.1. We observe that $\beta^{p} \leq \beta$, as the second term on the right-hand side of equation (2.25) is non-negative (in fact strictly positive for $b \neq r$ ). Second, when $p=$ -1 we have $\beta^{p}=\beta$, and for $p \in[-1 / 2,0), \beta^{p} \leq \Lambda$. Therefore, exceeding the growth maximizing strategy beyond a multiple denoted by $p \in[-1 / 2,0)$ makes the trend of the net worth process of an open economy even worse than the trend of a closed one. More clearly, a closed economy will be worse-off in terms of growth and risk level when opening to the rest of the world with a leverage strategy denoted by (2.23) with $p \in[-1 / 2,0)$.

\subsection{Survival Maximization}

In the previous section, we solved growth problems showing that the growth maximizing strategy minimizes the expected time to reach a goal and maximizes the expected survival time given the conditions of an economy. Via the solutions, we also devised a method to obtain an estimate on the time that the net worth process of a non-resilient economy will hit a lower boundary. While such information is useful for having a view on the happening of a possible debt crisis, another relevant objective would be finding the probability of the occurrence of the crisis. From the point of this view, we may therefore consider the probability of ruin, as leverage brings the possibility of default along with an extra source of risk coming from the level of the net effective rate of return on net foreign assets.

\footnotetext{
${ }^{12}$ Notice that we don't state the case for $\beta^{p}$ when $\boldsymbol{f}^{*} \leq \theta$, since the leverage strategy of a non-resilient economy is always larger than zero.
} 
Therefore, in this section, we will find the optimal leverage strategy that minimizes to probability of ruin of an economy.

One method to reduce the ruin probability is to pursue fractional growth maximizing strategy as that strategy diminishes the potential downside by reducing the risk taken for growth ${ }^{13}$. As we observe, from equation (2.23), fractional growth maximizing strategy corresponds to a risk aversion parameter value $p \in(-\infty,-1)$, where as $p \downarrow-\infty$, risk taking diminishes. However, the problem with pursuing the fractional growth strategy based on a risk aversion parameter value $p$ is that the choice on $p$ is a subjective (hence, arbitrary) task as there is a lack of connection between $p$ and the fundamentals of an economy. Therefore, an optimal leverage strategy needs to account for the fundamental factors of an economy as the risk aversion level in the economy may be affected by these factors. With this, we may also observe how the risk aversion level changes as the fundamental factors change in an economy. Especially, we may address the problem of why an economy in a survival mode (that is when $\beta<0$ ) would ever pursue multiples of growth maximizing leverage strategy.

Second, in the previous section, we observed that the growth maximizing leverage strategy is independent of the consumption rate; while we may observe the indirect effect of the leverage on consumption via the identity $C(t)=c X^{f}(t)$, we may not clearly see the effect of the consumption on the leverage strategy when it is a growth maximizing one. Moreover, even if the growth maximizing leverage strategy enhances growth, as long as the economy is in a survival mode, more leverage than the level designated by the growth maximizing leverage strategy has to be pursued in order to supply the consumption. In other words, as propensity of consumption increases leverage level increases (even beyond the level of the growth maximizing strategy). Therefore, establishing a relation between the risk aversion parameter and the fundamental factors of an economy may help us to measure the effect of consumption on the leverage level.

Finally, when opening to the rest of the world, an economy exposes itself to an extra source of risk arising from the volatility of the net effective rate of return on net foreign assets. While exposure under certain forms may help the economy to improve the growth of its net worth, it may also increase the possibility of ruin. A central problem is then to see the contrasting relationship between the growth and ruin minimization policies under changing economic environment. To this end, we set the objective in this section as maximizing the probability of reaching a higher net worth level $U$ without first hitting a net worth level $L$. In other words, the objective is maximizing the survival probability of an economy. In this form, the objective is equivalent to ruin probability minimization. As a result, we define the objective by

$$
F(x)=\sup _{f \in \mathcal{A}(x)} \mathbb{P}_{x}\left(\tau_{U}^{f}<\tau_{L}^{f}\right)
$$

\footnotetext{
${ }^{13}$ We refer the readers to Yener, Stengos \& Yazgan (2014) and the references therein for a further discussion on this subject.
} 
where $\mathbb{P}_{x}()=.\mathbb{P}(. \mid X(0)=x)$. Then, the results follow in the next proposition.

Proposition 2.2. Let the net worth process $\left\{X^{f}(t), 0 \leq t<\infty\right\}$ be the solution to the stochastic differential equation given in (2.11). Then, the optimal value function is given by

$$
F(x)=\frac{L^{1+\gamma_{+}}-x^{1+\gamma_{+}}}{L^{1+\gamma_{+}}-U^{1+\gamma_{+}}} \quad \text { for } x \in[L, U],
$$

where $\gamma_{+} \in(-\infty, 0) \backslash\{-1\}$ is

$$
\gamma_{+}= \begin{cases}\frac{\left[2 \sigma^{2}[(b-c)+(b-r) \alpha(\rho \kappa-1)]+\sqrt{\Delta}\right]}{2 \sigma^{2}\left[\sigma^{2}(\alpha(\rho \kappa-1))^{2}-\sigma_{2}^{2}\right]}, & \text { if } \theta<-\frac{1}{\gamma_{+}} \frac{b-r}{\sigma^{2}}+\alpha(\rho \kappa-1) ; \\ -\frac{2[(b-c)+\theta(b-r)]}{\sigma^{2}\left[\theta^{2}+\alpha+2 \theta(1-\rho \kappa)\right]}, & \text { if }-\frac{1}{\gamma_{+}} \frac{b-r}{\sigma^{2}}+\alpha(\rho \kappa-1) \leq \theta,\end{cases}
$$

with $\sigma^{2}(\alpha(\rho \kappa-1))^{2}-\sigma_{2}^{2}<0$ and the discriminant is given by

$$
\Delta=4 \sigma^{4}[(b-c)+(b-r) \alpha(\rho \kappa-1)]^{2}-4 \sigma^{2}\left[\sigma^{2}(\alpha(\rho \kappa-1))^{2}-\sigma_{2}^{2}\right](b-r)^{2} .
$$

Then, the optimal leverage strategy for $L<x<U$ is equal to

$$
\boldsymbol{f}^{*}= \begin{cases}-\frac{1}{\gamma_{+}} \frac{b-r}{\sigma^{2}}+\alpha(\rho \kappa-1), & \text { if } \theta<-\frac{1}{\gamma_{+}} \frac{b-r}{\sigma^{2}}+\alpha(\rho \kappa-1) ; \\ \theta, & \text { if }-\frac{1}{\gamma_{+}} \frac{b-r}{\sigma^{2}}+\alpha(\rho \kappa-1) \leq \theta .\end{cases}
$$

Proof. Please see Appendix B.

Substituting the optimal leverage strategy in (2.29) to the wealth process yields, for $t<\tau_{L}^{f^{*}} \wedge \tau_{U}^{f^{*}}$

$$
\begin{array}{r}
X^{*}(t)=x \exp \left\{\beta^{\gamma} t+\left(\frac{1}{\gamma_{+}} \frac{b-r}{\sigma^{2}}+\alpha(\rho \kappa-1)\right) \sigma_{1} W_{1}(t)\right. \\
\left.+\left(1-\frac{1}{\gamma_{+}} \frac{b-r}{\sigma^{2}}+\alpha(\rho \kappa-1)\right) \sigma_{2} W_{2}(t)\right\},
\end{array}
$$

where $\beta^{\gamma}$ is given similarly to $(2.25)$. That is, we have

$$
\beta^{\gamma}= \begin{cases}\beta-\frac{1}{2}\left(1+\frac{1}{\gamma_{+}}\right)^{2} \frac{(b-r)^{2}}{\sigma^{2}}, & \text { if } \theta<-\frac{1}{\gamma_{+}} \frac{b-r}{\sigma^{2}}+\alpha(\rho \kappa-1) \\ \Lambda+\sigma^{2} \theta\left[\frac{b-r}{\sigma^{2}}+\alpha(\rho \kappa-1)-\frac{1}{2} \theta\right], & \text { if }-\frac{1}{\gamma_{+}} \frac{b-r}{\sigma^{2}}+\alpha(\rho \kappa-1) \leq \theta\end{cases}
$$

From the results, we see that the optimal leverage strategy is similar to the specification of the power utility maximizing leverage strategy. That is, $\gamma_{+}$appears as a risk aversion parameter and is dependent on the underlying factors of an economy. From this dependence, we observe that the optimal leverage strategy is related to the level of the proportional consumption rate $c$. Therefore, while an economy maximizes growth regardless of the level of its consumption, it takes marginal propensity of consumption into 
consideration when determining the optimal leverage strategy for maximizing its survival probability. Furthermore, from the specification of the optimal leverage strategy in (2.29), we observe that $\gamma_{+}$and $\boldsymbol{f}^{*}$ is increasing in $c$. To see it more clearly, we write

$$
\frac{\partial \gamma_{+}}{\partial c}=\frac{1}{\left[\sigma_{2}^{2}-\sigma^{2}(\alpha(\rho \kappa-1))^{2}\right]}\left[1+\frac{1}{\sqrt{\Delta}} 2 \sigma^{2}[(b-c)+(b-r) \alpha(\rho \kappa-1)]\right] .
$$

We have $\sqrt{\Delta}>2 \sigma^{2}[(b-c)+(b-r) \alpha(\rho \kappa-1)]$. Therefore,

$$
\left|\frac{1}{\sqrt{\Delta}} 2 \sigma^{2}[(b-c)+(b-r) \alpha(\rho \kappa-1)]\right|<1,
$$

and $\left[\sigma_{2}^{2}-\sigma^{2}(\alpha(\rho \kappa-1))^{2}\right]>0$ as shown in the proof of the problem. As a result, $\frac{\partial \gamma_{+}}{\partial c}>0$. That is, assuming that other factors remain the same, as an economy consumes higher proportion of its net worth, it then exposes itself to more debt in order to finance increasing consumption level. As we have seen in the previous section, increasing consumption worsens the economic condition which might eventually lead to a downturn in the net worth process. However, while consumption may solely be enough to consider as a reason for economic downturn, there may also be other factors (i.e. increasing uncertainty in return on capital) that may also lead to downturn. In this case, then, checking the relationship between the economic conditions of an economy and its leverage under a broader approach is more sensible. To this end, we apply the analysis of Browne (1999) to our case and write the following corollary.

Corollary 2.2. Let $\gamma_{+}$be given by (2.28). Then, we have

$$
\beta\left\{\begin{array}{lll}
<0 & \text { if } \quad \gamma_{+} \in(-1,0) \\
=0 & \text { if } \quad \gamma_{+}=-1 \\
>0 & \text { if } \quad \gamma_{+} \in(-\infty,-1) .
\end{array}\right.
$$

Proof. Please see appendix C.

From the above corollary and (2.31), we observe that $\beta^{\gamma}<0$ whenever $\beta<0$. In this case, the economy will be in a bad condition and gets extremely bad as $\gamma_{+}$is closer to zero. Then, the exposure to debt for maximizing the survival probability will be higher than it is for maximizing the expected survival time. Thus, as the economic conditions worsen, debt exposure for maximizing the survival probability increases above a level needed for maximizing the expected survival time.

On the other hand, when $\beta>0, \beta^{\gamma}$ is either negative or positive. If $\beta^{\gamma}<0$, the economy is still in a bad condition, but not as bad. Then, the exposure to debt for maximizing the survival probability will be lower than the one for maximizing the expected survival time. The aforementioned conclusion is similar when $\beta^{\gamma}>0$. However, notice that the value of $\beta^{\gamma}$ increases as $\gamma_{+}$decreases. Thus, when the factors of the economy 
leads to a decline in $\gamma_{+}$and improvement in the economic conditions, the exposure to debt diminishes below a level denoted by the growth maximizing strategy. In other words, as the economic conditions improve, in order to maximize its survival probability, an economy will eventually be less exposed to debt than it should be when minimizing the expected time to achieve a goal.

In sum, applying the analysis of Browne (1999) further to our case, bad economic conditions lead to bold leverage strategy values for the survival probability maximization. In other words, under bad economic regimes excessive risk may be taken at the expense of the growth when it comes to survival probability maximization. On the other hand, good economic conditions lead to timid leverage strategy values for the survival probability maximization. Since the trend is to the advantage of the economy, then excessive risk taking must be avoided in order to stay on course.

Next, we would like to analyse the conditions under which exposure to debt is better or worse than no exposure at all when maximizing the survival probability. To this end, we first provide the following corollary that will be used in the analysis. Its proof can be done by setting $\boldsymbol{f}=0$ in the first part of the proof of the problem under consideration.

Corollary 2.3. With no exposure to debt, the probability of reaching a higher net worth without first hitting a lower one is equal to

$$
F(x)=\frac{L^{1+\bar{\gamma}}-x^{1+\bar{\gamma}}}{L^{1+\bar{\gamma}}-U^{1+\bar{\gamma}}} \quad \text { for } x \in[L, U]
$$

where $\bar{\gamma}$ is

$$
\bar{\gamma}=-\frac{2(b-c)}{\sigma_{2}^{2}}
$$

From the specification (2.36), we see that the sign of $\bar{\gamma}$ depends on the sign of $b-c$. It is negative when the mean rate of return on capital is higher than the proportional rate of consumption. In addition, as we showed in appendix $\mathrm{D}, F(x)$ is a monotonically decreasing function in $\gamma$. Therefore, its value is higher as $b-c$ is higher and $\sigma_{2}$ is lower. Especially, given the specification of $\Lambda$ in equation (2.13), we see that higher $b-c$ and lower $\sigma_{2}$ implies higher values for $\Lambda$, hence better self-sufficiency for an economy.

First, we consider the case when $b=r$. Under this equality, the optimal strategy becomes equal to $\alpha(\rho \kappa-1)$. In this case, the value of $\gamma_{+}$becomes

$$
\gamma_{+}=-\frac{2(b-c)}{\left[\sigma_{2}^{2}-\sigma^{2}(\alpha(\rho \kappa-1))^{2}\right]}
$$

The denominator is positive. We need $b-c>0$, so that we can have $\gamma_{+}<0$ otherwise the maximization problem would not hold. That is, if the mean rate of return on capital is equal to the mean net effective rate of return on net foreign assets, an economy can maximize its survival probability as long as it keeps its consumption below the mean rate 
of return on capital. From the above value we can deduce that an exposure amount of $\alpha(\rho \kappa-1)$ maximizes the survival probability. In this case, both $\gamma_{+}$and $\bar{\gamma}$ are negative, but, $\gamma_{+}<\bar{\gamma}$ because $\left[\sigma_{2}^{2}-\sigma^{2}(\alpha(\rho \kappa-1))^{2}\right]<\sigma_{2}^{2}$. In addition, we have $F(x)$ monotonically decreasing in $\gamma$. Therefore, exposure to debt is better than no exposure at all.

Second, when $b \neq r$ with $b-c<0$, exposure to debt is good because $\gamma_{+}<0<\bar{\gamma}$. However, the result is ambiguous when $b-c>0$, because both $\gamma_{+}$and $\bar{\gamma}$ are negative, and their order on the negative axis of the real line is not clear. Therefore, when the mean rate of return on capital is higher than the proportional consumption rate, debt exposure may not necessarily be a better strategy than no exposure at all in order to maximize survival probability. It then follows that, as the self-sufficiency in an economy lessens, pursuit of bold policies may become more likely in order to finance excess consumption. On the other hand, timid policies are pursued as an economy becomes more self-sufficient. Especially, if the underlying factors are right, no exposure to debt might even be a better policy for a self-sufficient economy.

Finally, as we did in the previous section, we use again the power utility maximizing strategy in (2.23) to represent the actual leverage strategy in order to calculate the survival probability of an economy. Under such representation we write the following corollary.

Corollary 2.4. The survival probability under the leverage strategy given in equation (2.23) is equal to

$$
F(x)=\frac{L^{1+\alpha}-x^{1+\alpha}}{L^{1+\alpha}-U^{1+\alpha}} \quad \text { for } x \in[L, U],
$$

where $\alpha$ is

$$
\alpha=\frac{-2\left[(b-c)+(b-r)\left(-\frac{1}{p} \frac{b-r}{\sigma^{2}}+\alpha(\rho \kappa-1)\right)\right]}{\frac{1}{p^{2}} \frac{(b-r)^{2}}{\sigma^{2}}+\sigma_{2}^{2}}
$$

Proof. The proof of this corollary is similar to the proof of the corollary 2.1. To this end, we set $\boldsymbol{f}$ in the associated Hamilton-Jacobi-Bellman equation of this problem equal to the strategy that maximizes the power utility, then solve for the function that is a solution of this Dirichlet-type problem.

\section{The Data \& Analysis}

\subsection{The Data \& Its Estimation}

For the application, we consider Greece, because the country has been the point of attention of the economics and finance community for a while due its surging debt problems. To this end, we collect data and estimate the parameters as in Yener, Stengos \& Yazgan (2014). That is, five key variables of the net worth process ${ }^{14}$ are considered for the period

\footnotetext{
${ }^{14}$ These are capital stock, debt stock, consumption, investments, and gross domestic product.
} 
from 1980 to 2007 and they are all denominated in US dollars. The capital stock data, retrieved from the Fred Economic Data website, are based on the estimations of Feenstra and Timmer (2013). Furthermore, for investments we use the gross capital formation data obtained from the World Bank's World Development Indicators database, while for consumption we use the final consumption expenditure data from the same resource.

On the other hand, for the debt variable, as mentioned earlier, we use the net foreign assets data retrieved from the updated and extended version of the External Wealth of Nations Mark II database developed by Lane and Milesi-Ferretti (2007). Note that the estimation of the net effective rate of return $r(t)$ is done via the discretization procedure shown in Yener, Stengos \& Yazgan (2014), because the data for the net foreign asset is given annually. Furthermore, we refer the readers to Yener, Stengos \& Yazgan (2014) again for the estimation of the return on capital, $b(t)$, the proportional consumption rate, the mean values of the return on capital and the net effective rate of return. Note that in the paper we dropped the time index to simplify the notation and computations without loss of generality. However, we use deterministic consumption rate and mean values for the analysis. Once, we obtain the estimates for the key parameters, we then proceed to compute the values of interest to predict the Greek debt crisis and analyse the underlying reasons.

Before starting the analysis, we remind the readers that if the non-resilience condition, which is $\beta_{t}<0$ and $\mathbf{f}(t)-\mathbf{f}^{*}(t)>0$, is coupled with prolonged and persistent deterioration in fundamentals and increasing excessive leverage, it indicates a possible debt crisis. Therefore, as we did in Yener, Stengos \& Yazgan (2014), we first bring the realized leverage of Greece to the attention in the graph here below. As we observe, Greece was a debtor nation for the whole period and its realized leverage increased from the order of $1.6 \%$ in 1996 to $58.5 \%$ in 2007; a substantial increase that eventually led to Greek debt crisis.

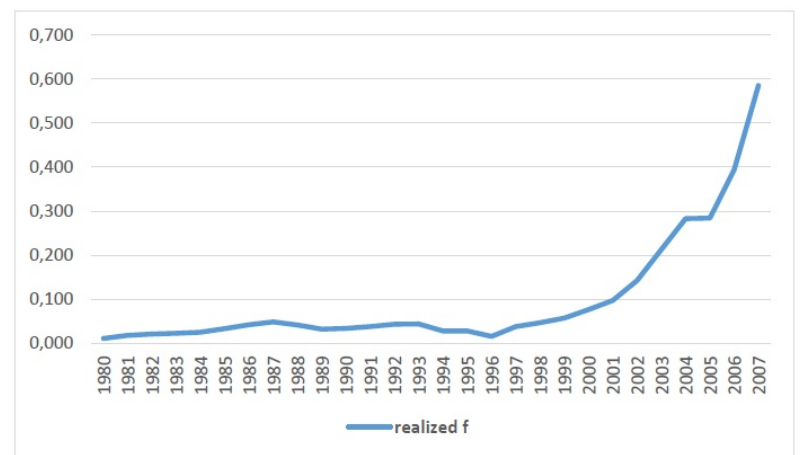

Fig. 1: Realized Leverage Levels of Greece

\subsection{The Numerical Analysis}




\subsubsection{Prediction of the Debt Crisis in Greece}

As previously explained in Yener, Stengos \& Yazgan (2014), the non-resilience condition in Greece was first met in around year 2006. From figure 2, we observe that there was a steady decline in $\beta$, while an increase in the realized leverage to around $60 \%$ of the net worth (see figure 1). Especially, we also observe from figure 2 that the realized leverage eventually went above the growth maximizing leverage level around 2006 with negative $\beta$ during the same year. Therefore, deteriorating fundamentals eventually led to the realization of the non-resilience condition, which may therefore be considered as an early warning signal of a possible debt crisis in Greece.

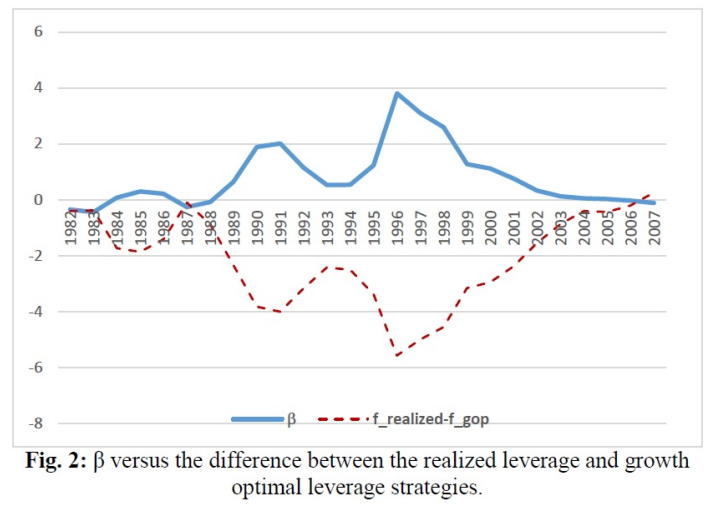

Given the indication of a possible debt crisis in Greece, we then proceed to estimate the expected time the net worth level hits a lower boundary level by using equation (2.17), as with $\beta<0$, Greece becomes a survival time maximizing nation. In figure 3 below, we provide the expected time estimates on the $\mathrm{y}$-axis for a given level of percentage decline of the net worth process from its 2007 value on the x-axis. Mainly, since a decline in the net worth may eventually lead to a debt crisis, we may then interpret the values in the $\mathrm{y}$-axis of figure 3 as the time to a possible sovereign default. To see if the estimates are appropriate, we need to obtain how the net worth of Greece changed after 2007 till the end of the crisis year (which is 2009). To this end, from the numbers provided in table $1^{15}$ , we first estimate the net foreign assets of Greece for each subsequent year by adding the current account deficits of Greece to 2007 value of Greece's net foreign assets ${ }^{16}$. Then, by using the capital stock data retrieved from the Fred Economic Data website for the date between 2007 and 2009, we find the net worth of Greece up until the end of the crisis year and calculate the drop percentage via $\ln \left(X\left(t_{1}\right) / X\left(t_{2}\right)\right)$ for $t_{1}=2007$ and $t_{2}=2009$, which

\footnotetext{
${ }^{15}$ Note that the numbers reported here are rounded to ease exposition.

${ }^{16}$ The net foreign assets data developed by Lane and Milesi-Ferretti (2007) were available up to 2007. Therefore, we need to estimate the net foreign asset values until the end of crisis year by adding the current account deficits of Greece that was calculated by using the percentage expression with regards to GDP. We retrieved the data from the World Bank's World Development Indicators database.
} 
gives around $6.15 \%$. We see from figure 3 that for such drop percentage, the estimated default time is 6 months from the end of 2007 with an upper bound of 20 months, showing that the prediction captures the time of debt crisis somewhat closely.

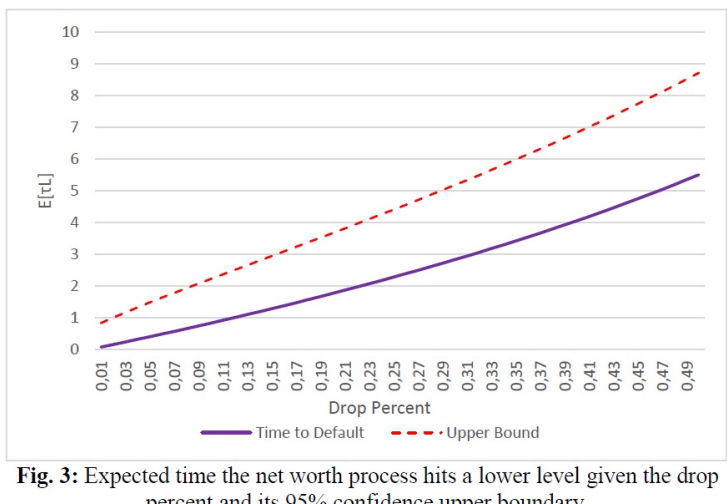

percent and its $95 \%$ confidence upper boundary.

Table 1: Data to Estimate Default Time

\begin{tabular}{ccccc}
\hline \hline Year & $\begin{array}{c}\text { Current Acct. Bal. } \\
\text { (\% of GDP) }\end{array}$ & $\mathrm{K}(\mathrm{t})$ & $\mathrm{L}(\mathrm{t})$ & $\mathrm{X}(\mathrm{t})$ \\
\hline 2007 & - & $911,231,812,500$ & $324,267,396,512$ & $586,964,415,988$ \\
2008 & $-14.469 \%$ & $932,051,312,500$ & $363,354,155,800$ & $568,697,156,700$ \\
2009 & $-10.888 \%$ & $943,432,187,500$ & $391,473,538,654$ & $551,958,648,846$ \\
\hline
\end{tabular}

Given our method for indicating and predicting a possible debt crisis, we then proceed to apply the results we obtained under the survival probability maximization problem for the analysis of Greek economy through the period of study considered in the paper.

\subsubsection{Analysis of Leverage Policies}

Next, we compare the realized, growth maximizing, and survival probability maximizing leverage strategies to understand further the seeds of the debt crisis in Greece. Through this comparison, our aim is also to make a better sense of the analyses of the results provided in section 2.3. First, we observe from figure 4 that when the factors of Greek economy lead to good economic conditions (that is, $\beta>0$ ), survival probability maximizing leverage strategy values turned out to be lower than the growth maximizing leverage strategy values. This follows from our previous finding that under good economic conditions pursuing timid strategy for survival probability maximization is better. 


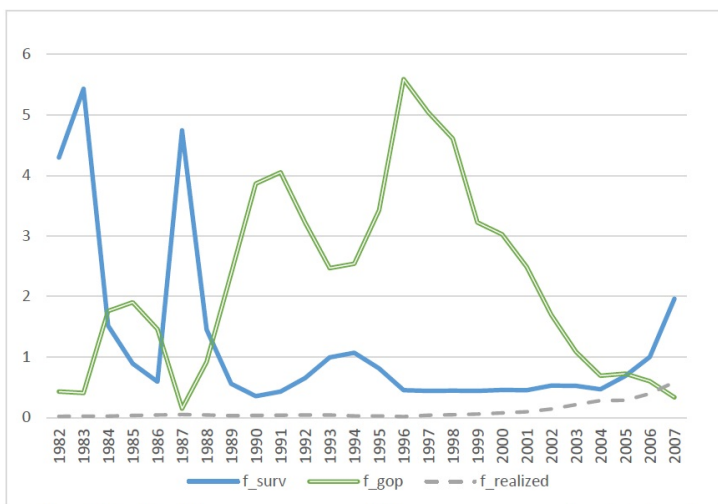

Fig. 4: Realized leverage strategy of Greece versus growth optimal and survival probability maximizing strategies.

However, we observe a more steady path for the survival probability maximizing leverage strategy and a consistent deteriorating path for the growth maximizing strategy after around year 1996 and until 2005 in figure 4 again. Especially, after 2005, the model offers bold strategy for the survival probability maximization as the factors of the economy started to deteriorate. This means more leverage to deal with the bad effects of the downturn. In reality, though it wasn't as high as high the probability maximizing leverage strategy suggests, the realized leverage in Greece did indeed started to increase from 1996, and it did at a rate much higher after 2004.

Nevertheless, throughout the analysis period the survival maximizing leverage strategy is distinctly higher than the realized leverage strategy and this difference, as we observe from figure 5, did indeed led to higher survival probability under the optimal strategy until around 2004. On the other hand, we see that the survival probability values related to optimal and realized leverage strategies after year 2004 are much closer to each other although the gap in between the pertaining leverage strategies depicted in figure 4 is wide. Therefore, the distinction between the effect of the optimal and the realized strategies over the value of the survival probability fades away after year 2004.

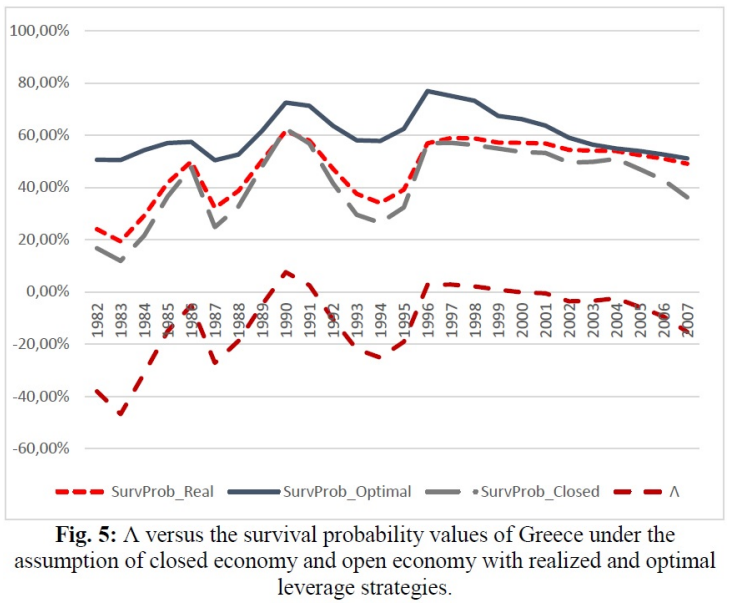


Finally, we see from the path of $\Lambda^{17}$ in figure 5 that the self-sufficiency of Greek economy followed a fluctuating path in between the positive and negative values throughout the observation period. The relevant part for our analysis is the deterioration that started in year 1996 and became negative again in around 2001. Especially, after 2004, the deterioration became much steeper. Adding the associated decline in the growth maximizing leverage strategy and increase in the realized strategy (see figure 4) as well, we may say that the growth in Greece was hampered, and the country needed to resort to external resources to meet the excess demand in its economy; hence the use of foreign debt for non-productive resources. Even Greece were to borrow optimally, as evident from figure 5, it would still face a downward sloped survival probability trend after 1996 due to its increasing lack of self-sufficiency. Furthermore, note that the attenuation in self-sufficiency was steeper after 2004, exactly the time when the expansionary policy in Greece became steeper. As a result, expansionary strategies with increasing lack of self-sufficiency was certainly a sign of the crisis that followed; hence, excessive risk taking at the expense of growth under bad economic conditions (see the analysis of proposition 2.2 in section 2.3).

\section{Conclusion}

In this paper, we created a model driven by uncertainty in return on capital and net effective rate of return on net foreign assets. The model is the same as the one introduced in Fleming and Stein (2004), however, the proportional consumption rate in our case is considered exogenous. We then used the techniques of stochastic optimal control theory in order to solve two problems that are related to maximizing growth and survival probability. In the first problem, we solve for the minimum (maximum) expected time to reach a goal (to survive). In the second problem, we find the maximum survival probability, which is defined as the probability that the net worth process reaches a higher net worth without first hitting a lower one.

By using the results found under the first problem, we then show that the growth maximizing leverage strategy, in addition to its growth enhancing property, improves both the goal reaching and survival times of an economy. Furthermore, we also provide the closed form solution for the estimated default time of a non-resilient economy. In the second problem, on the other hand, we show that the survival maximizing probability is similar to the specification of a power utility maximizing strategy as it includes a risk aversion parameter $\gamma_{+} \in(-\infty, 0) \backslash\{-1\}$ dividing the term $(b-r) / \sigma^{2}$. Through this parameter, the risk aversion becomes dependent on the factors of the economy and the leverage strategy becomes, in turn, dependent on the proportional consumption rate $c$. Given this dependence, we established that the exposure to debt increases with increasing

\footnotetext{
${ }^{17}$ We remind the reader that $\Lambda$ was the trend of the net worth of a closed economy. Therefore, we use it as the measure of the self-sufficiency of an economy.
} 
consumption, and it is likely to happen in the form of borrowing.

We also showed in solving the second problem that while bad economic conditions lead to bold strategies at the expense of growth, good economic conditions lead to timid strategies when maximizing the survival probability. Therefore, as long as an economy is in a bad condition, then, the survival probability maximizing leverage level in that economy may be higher than the growth maximizing leverage strategy. In fact, we show that an economy exposes itself to debt whenever $b \neq r$ with $b-c<0$, implying that as an economy becomes less self-sufficient, pursuit of bold strategies becomes possible in order to finance excess consumption. Finally, we applied the results for the analysis of the recent debt crisis in Greece. Via our application, we saw that we manage to predict the debt crisis in Greece somewhat closely and that the underlying reason behind the crisis was the expansionary policy with declining self-sufficiency of the country.

\section{Appendix}

\section{A Proof of Proposition 2.1}

The proof of the maximisation problem is given first. The proof of the minimization problem is omitted as it is similar to the proof of the maximization problem ${ }^{18}$.

We let $\underline{F}(x)$ be the function given by $(2.17)$. We see that $\underline{F}(x)$ is a $C^{2}((L, \infty))$ function with $\underline{F}_{x}>0, \underline{F}_{x x}<0^{19}$ and continuous on $[L, \infty)$. Then, the associated Hamilton-JacobiBellman (HJB, hereafter) equation is

$1+(b-c) x \underline{F}_{x}+\sup _{\boldsymbol{f}}\left\{\boldsymbol{f}(b-r) x \underline{F}_{x}+\frac{1}{2}\left[\boldsymbol{f}^{2} \sigma_{1}^{2}+(1+\boldsymbol{f})^{2} \sigma_{2}^{2}-2 \boldsymbol{f}(1+\boldsymbol{f}) \rho \sigma_{1} \sigma_{2}\right] x^{2} \underline{F}_{x x}\right\}=0$

subject to the boundary condition $\underline{F}(L)=1$. From the first order conditions in(A.1), we let the maximizer $\boldsymbol{f}^{*}$ satisfy

$$
f^{*}(x)= \begin{cases}-\frac{b-r}{\sigma^{2}} \frac{\underline{F}_{x}}{x \underline{F}_{x x}}+\alpha(\rho \kappa-1), & \text { if } \theta<-\frac{b-r}{\sigma^{2}} \frac{\underline{F}_{x}}{x \underline{F}_{x x}}+\alpha(\rho \kappa-1) \\ \theta, & \text { if }-\frac{b-r}{\sigma^{2}} \frac{\underline{F}_{x}}{x \underline{F}_{x x}}+\alpha(\rho \kappa-1) \leq \theta\end{cases}
$$

where, as previously denoted, $\sigma^{2}=\sigma_{1}^{2}+\sigma_{2}^{2}-2 \rho \sigma_{1} \sigma_{2}, \alpha=\sigma_{2}^{2} / \sigma^{2}$, and $\kappa=\sigma_{1} / \sigma_{2}$. We

\footnotetext{
${ }^{18}$ Note that to find the optimal results in all problems we use the dynamic programming principle (see for example Fleming and Soner (1993) and Björk (2004)).

${ }^{19}$ We hide the arguments of the functions when necessary in order to simplify the notation.
} 
substitute (A.2) into (A.1) to obtain the non-linear partial differential equations

$$
\left\{\begin{array}{c}
1+[(b-c)+(b-r) \alpha(\rho \kappa-1)] x \underline{F}_{x}-\frac{1}{2} \frac{(b-r)^{2}}{\sigma^{2}} \frac{\underline{F}_{x}^{2}}{\underline{F}_{x x}}-\frac{1}{2}\left[\sigma^{2}(\alpha(\rho \kappa-1))^{2}-\sigma_{2}^{2}\right] x^{2} \underline{F}_{x x}=0, \\
\text { if } \theta<-\frac{b-r}{\sigma^{2}} \frac{\underline{F}_{x}}{x \underline{F_{x x}}}+\alpha(\rho \kappa-1) \\
1+[(b-c)+\theta(b-r)] x \underline{F}_{x}+\frac{1}{2}\left[\theta \sigma^{2}(\theta-2 \alpha(\rho \kappa-1))+\sigma_{2}^{2}\right] x^{2} \underline{F}_{x x}=0, \\
\text { if }-\frac{b-r}{\sigma^{2}} \frac{\underline{F}_{x}}{x \underline{F}_{x x}}+\alpha(\rho \kappa-1) \leq \theta .
\end{array}\right.
$$

By substituting $\underline{F}(x)$ into (A.3) (and also by considering (2.16)), we observe that the equalities in the system of equations above are satisfied. Then, the maximizer is equal to

$$
\boldsymbol{f}^{*}= \begin{cases}\frac{b-r}{\sigma^{2}}+\alpha(\rho \kappa-1), & \text { if } \theta<\frac{b-r}{\sigma^{2}}+\alpha(\rho \kappa-1) ; \\ \theta, & \text { if } \frac{b-r}{\sigma^{2}}+\alpha(\rho \kappa-1) \leq \theta .\end{cases}
$$

Verification: We start by substituting the maximizer into the net worth process. The wealth process becomes for $t<\tau_{L}^{f^{*}}$

$X^{*}(t)=x \exp \left\{\beta t-\left(\frac{b-r}{\sigma^{2}}+\alpha(\rho \kappa-1)\right) \sigma_{1} W_{1}(t)+\left(1+\frac{b-r}{\sigma^{2}}+\alpha(\rho \kappa-1)\right) \sigma_{2} W_{2}(t)\right\}$.

We are verifying for the maximisation problem. Therefore, we have $\beta<0$ and $\mathbb{P}\left(X^{*}(t) \geq\right.$ $L) \rightarrow 0$ as $t \rightarrow \infty$. This implies $\tau_{L}^{f^{*}}<\infty$ almost surely. Then, we have

$$
\int_{0}^{\tau_{L}^{f^{*}}}\left(\boldsymbol{f}^{*}\left(X^{*}(s)\right)\right)^{2} d s=\left(\frac{b-r}{\sigma^{2}}+\alpha(\rho \kappa-1)\right)^{2} \tau_{L}^{f^{*}}<\infty
$$

almost surely. It follows that $\boldsymbol{f}^{*} \in \mathcal{A}(x)$. Next, we fix $x \in(L, \infty)$ and introduce the function

$$
M\left(t, X^{f}(t)\right)=\underline{F}\left(X^{f}(t)\right)+t .
$$

Note that $M(0, x)=\underline{F}(x)$. In addition, we define the stopping time

$$
\tau_{n}^{f}=\tau_{L}^{f} \wedge \inf \left\{t>0 \mid \int_{0}^{t} f^{2}\left(X^{f}(s)\right) d s=n\right\} \quad \text { for } \quad n \in \mathbb{N} .
$$

Then, we choose an arbitrary strategy $f \in \mathcal{A}(x)$, and from Itô's formula we write by inserting $X^{f}(\cdot)$ into function $\underline{F}$

$$
\begin{aligned}
M\left(\tau_{n}^{f}, X^{f}\left(\tau_{n}^{f}\right)\right)= & \underline{F}(x)+\tau_{n}^{f}+\int_{0}^{\tau_{n}^{f}}\left\{\mathcal{L}^{f} \underline{F}\left(X^{f}(s)\right)\right\} d s \\
& -\int_{0}^{\tau_{n}^{f}} X^{f}(s) \boldsymbol{f}\left(X^{f}(s)\right) \sigma_{1} \underline{F}_{x}\left(X^{f}(s)\right) d W_{1}(s) \\
& +\int_{0}^{\tau_{n}^{f}} X^{f}(s)\left(1+\boldsymbol{f}\left(X^{f}(s)\right)\right) \sigma_{2} \underline{F}_{x}\left(X^{f}(s)\right) d W_{2}(s) .
\end{aligned}
$$


When the conditional expectation of the above terms are taken, the stochastic integrals vanish because

$$
\begin{aligned}
& \mathbb{E}_{x}\left[\int_{0}^{\tau_{n}^{f}}\left(X^{f}(s) \boldsymbol{f}\left(X^{f}(s)\right) \sigma_{1} \underline{F}_{x}\left(X^{f}(s)\right)\right)^{2} d s\right] \\
\leq & \sigma_{1}^{2} \frac{1}{\beta^{2}} \mathbb{E}_{x}\left[\int_{0}^{\tau_{n}^{f}} \boldsymbol{f}^{2}\left(X^{f}(s)\right) d s\right] \\
\leq & \sigma_{1}^{2} \frac{1}{\beta^{2}} n<\infty
\end{aligned}
$$

and

$$
\begin{aligned}
& \mathbb{E}_{x}\left[\int_{0}^{\tau_{n}^{f}}\left(X^{f}(s) \sigma_{2} \underline{F}_{x}\left(X^{f}(s)\right)\right)^{2} d s\right] \\
\leq & \sigma_{2}^{2} \frac{1}{\beta^{2}}<\infty .
\end{aligned}
$$

Then, we have from (A.9) and (A.10)

$$
\mathbb{E}_{x}\left[\int_{0}^{\tau_{n}^{f}}\left(X^{f}(s)\left(1+\boldsymbol{f}\left(X^{f}(s)\right)\right) \sigma_{2} \underline{F}_{x}\left(X^{f}(s)\right)\right)^{2} d s\right]<\infty
$$

We saw in the first part that $\underline{F}(x)$ solves the HJB equation. Then, $\forall \boldsymbol{f} \in \mathbb{R}$ and for each $s$ we have $\mathbb{P}$-a.s.

$$
1+\mathcal{L}^{f} \underline{F}\left(X^{f}(s)\right) \leq 0
$$

As a result, we obtain the inequality

$$
\underline{F}(x) \geq \mathbb{E}_{x}\left[M\left(\tau_{n}^{f}, X^{f}\left(\tau_{n}^{f}\right)\right)\right] .
$$

The function $\underline{F}(x) \leq \frac{1}{|\beta|} \ln (x / L)+K_{1}<\infty$ for some constant $K_{1} \geq 0$. In addition, we have as $n \rightarrow \infty, \tau_{n}^{f} \rightarrow \tau_{L}^{f}$. Therefore, from the dominated convergence theorem, as $n \rightarrow \infty$

$$
\mathbb{E}_{x}\left[M\left(\tau_{n}^{f}, X^{f}\left(\tau_{n}^{f}\right)\right)\right] \rightarrow \mathbb{E}_{x}\left[M\left(\tau_{L}^{f}, X^{f}\left(\tau_{L}^{f}\right)\right)\right] .
$$

Thus, the inequality in (A.13) becomes

$$
\begin{aligned}
\underline{F}(x) & \geq \mathbb{E}_{x}\left[M\left(\tau_{L}^{f}, X^{f}\left(\tau_{L}^{f}\right)\right)\right] \\
& =\mathbb{E}_{x}\left[\tau_{L}^{f}\right]
\end{aligned}
$$


When the supremum is taken over all admissible strategies we obtain

$$
\underline{F}(x) \geq \sup _{f \in \mathcal{A}(x)} \mathbb{E}_{x}\left[\tau_{L}^{f}\right]=\underline{F}(x)
$$

In fact, when $\boldsymbol{f}^{*}$ is chosen one could check from (A.9) and (A.11) that the stochastic integral vanishes. With this strategy, we have for each $s$ and $\mathbb{P}$-a.s.

$$
1+\mathcal{L}^{f^{*}} \underline{F}\left(X^{*}(s)\right)=0 .
$$

Thus, the inequality in (A.15) becomes equality and we obtain

$$
\underline{F}(x)=\mathbb{E}_{x}\left[\tau_{L}^{f^{*}}\right]
$$

showing that $f^{*}(x)$ is the optimal strategy and $\underline{F}(x)$ is the optimal value function.

The minimisation problem can be proved in a similar way. However, we need to take into account that the inequality in (A.12) should be reversed. Then, the results follow similarly.

\section{B Proof of Proposition 2.2}

We start by assuming that $F(x)$ is $C^{2}((L, U))$ with $F_{x}>0, F_{x x}<0$, and $F:[L, U] \rightarrow$ $[0,1]$. Then, the associated HJB equation for the problem is

$$
(b-c) x F_{x}+\sup _{f}\left\{\boldsymbol{f}(b-r) x F_{x}+\frac{1}{2}\left[\boldsymbol{f}^{2} \sigma_{1}^{2}+(1+\boldsymbol{f})^{2} \sigma_{2}^{2}-2 \boldsymbol{f}(1+\boldsymbol{f}) \rho \sigma_{1} \sigma_{2}\right] x^{2} F_{x x}\right\}=0,
$$

subject to the boundary conditions $F(L)=0$ and $F(U)=1$. From the first order conditions in (B.1), we find the maximizer $\boldsymbol{f}^{*}$ as

$$
f^{*}(x)= \begin{cases}-\frac{b-r}{\sigma^{2}} \frac{F_{x}}{x F_{x x}}+\alpha(\rho \kappa-1), & \text { if } \theta<--\frac{b-r}{\sigma^{2}} \frac{F_{x}}{x F_{x x}}+\alpha(\rho \kappa-1) ; \\ \theta, & \text { if }-\frac{b-r}{\sigma^{2}} \frac{F_{x}}{x F_{x x}}+\alpha(\rho \kappa-1) \leq \theta\end{cases}
$$

Then, by substituting (B.2) into (B.1), we obtain the non-linear partial differential equations to obtain the non-linear partial differential equations

$$
\left\{\begin{array}{c}
{[(b-c)+(b-r) \alpha(\rho \kappa-1)] x F_{x}-\frac{1}{2} \frac{(b-r)^{2}}{\sigma^{2}} \frac{F_{x}^{2}}{F_{x x}}-\frac{1}{2}\left[\sigma^{2}(\alpha(\rho \kappa-1))^{2}-\sigma_{2}^{2}\right] x^{2} F_{x x}=0,} \\
\text { if } \theta<-\frac{b-r}{\sigma^{2}} \frac{F_{x}}{x F_{x x}}+\alpha(\rho \kappa-1) ; \\
{[(b-c)+\theta(b-r)] x F_{x}+\frac{1}{2}\left[\theta \sigma^{2}(\theta-2 \alpha(\rho \kappa-1))+\sigma_{2}^{2}\right] x^{2} F_{x x}=0,} \\
\text { if }-\frac{b-r}{\sigma^{2}} \frac{F_{x}}{x F_{x x}}+\alpha(\rho \kappa-1) \leq \theta .
\end{array}\right.
$$


To solve the above equation we guess a solution of the form $A_{1}-A_{2} x^{1+\gamma}$ where $A_{1}$ and $A_{2}$ are constants. By using this solution along with the boundary conditions we find the value function as

$$
F(x)=\frac{L^{1+\gamma}-x^{1+\gamma}}{L^{1+\gamma}-U^{1+\gamma}} .
$$

Next, we substitute (B.4) into (B.3), and for the first case, obtain the quadratic equation after rearranging the terms

$$
\gamma^{2} \sigma^{2}\left[\sigma^{2}(\alpha(\rho \kappa-1))^{2}-\sigma_{2}^{2}\right]-2 \gamma \sigma^{2}[(b-c)+(b-r) \alpha(\rho \kappa-1)]+(b-r)^{2}=0 .
$$

By solving the above equation we find the value of the roots of $\gamma$ as

$$
\begin{aligned}
& \gamma_{+}=\frac{1}{2 \sigma^{2}\left[\sigma^{2}(\alpha(\rho \kappa-1))^{2}-\sigma_{2}^{2}\right]}\left[2 \sigma^{2}[(b-c)+(b-r) \alpha(\rho \kappa-1)]+\sqrt{\Delta}\right] \\
& \gamma_{-}=\frac{1}{2 \sigma^{2}\left[\sigma^{2}(\alpha(\rho \kappa-1))^{2}-\sigma_{2}^{2}\right]}\left[2 \sigma^{2}[(b-c)+(b-r) \alpha(\rho \kappa-1)]-\sqrt{\Delta}\right],
\end{aligned}
$$

where the discriminant is equal to

$$
\Delta=4 \sigma^{4}[-((b-c)+(b-r) \alpha(\rho \kappa-1))]^{2}-4 \sigma^{2}\left[\sigma^{2}(\alpha(\rho \kappa-1))^{2}-\sigma_{2}^{2}\right](b-r)^{2} .
$$

Notice that the term $\left[\sigma^{2}(\alpha(\rho \kappa-1))^{2}-\sigma_{2}^{2}\right]$ is negative since $\rho \leq 1<1 / \kappa[1+1 / \sqrt{\alpha}$. Then, the discriminant has a real solution. Furthermore, $\gamma_{+} \gamma_{-}<0$. Therefore, the roots are of the different sign. We need $\gamma<0$ in order to have $F_{x}>0$ and $F_{x x}<0$. This is achieved by selecting $\gamma_{+}$. As a result, the maximizer under the first case is

$$
\boldsymbol{f}^{*}(x)=-\frac{1}{\gamma_{+}} \frac{b-r}{\sigma^{2}}+\alpha(\rho \kappa-1) .
$$

On the other hand, after the substitution of (B.4) into the second equation in (B.3), we obtain the value of $\gamma$ as given in second case of equation (2.28) in proposition 2.2.

Verification: We see that $F(x)$ is $C^{2}((L, U))$ with $F_{x}>0, F_{x x}<0$ and $F:[L, U] \rightarrow[0,1]$. The boundary conditions are satisfied as well. Next, we substitute the maximizer into the net worth process to obtain for $t<\tau_{L}^{f^{*}} \wedge \tau_{U}^{f^{*}}$

$$
\begin{array}{r}
X^{*}(t)=x \exp \left\{\beta^{\gamma} t+\left(\frac{1}{\gamma_{+}} \frac{b-r}{\sigma^{2}}+\alpha(\rho \kappa-1)\right) \sigma_{1} W_{1}(t)\right. \\
\left.+\left(1-\frac{1}{\gamma_{+}} \frac{b-r}{\sigma^{2}}+\alpha(\rho \kappa-1)\right) \sigma_{2} W_{2}(t)\right\} .
\end{array}
$$

We see that when $\beta^{\gamma}>0, \mathbb{P}\left(X^{*}(t) \geq U\right) \rightarrow 1$ as $t \rightarrow \infty$, and when $\beta^{\gamma}<0, \mathbb{P}\left(X^{*}(t) \geq L\right) \rightarrow$ 0 as $t \rightarrow \infty$. That is, $\tau_{L}^{f^{*}}<\infty$ and $\tau_{U}^{f^{*}}<\infty$ almost surely. Therefore, we have

$$
\int_{0}^{\tau_{L}^{f^{*}} \wedge \tau_{U}^{f^{*}}}\left(\boldsymbol{f}^{*}\left(X^{*}(s)\right)\right)^{2} d s=\left(-\frac{1}{\gamma_{+}} \frac{b-r}{\sigma^{2}}+\alpha(\rho \kappa-1)\right)^{2}\left(\tau_{L}^{f^{*}} \wedge \tau_{U}^{f^{*}}\right)<\infty
$$


almost surely, giving us $\boldsymbol{f}^{*} \in \mathcal{A}(x)$. Next, we fix a point $x \in(0, L)$ and introduce

$$
\tau^{*}=\tau_{L}^{f} \wedge \tau_{U}^{f},
$$

along with

$$
\tau_{n}^{f}=\tau^{*} \wedge \inf \left\{t>0 \mid \int_{0}^{t} f^{2}\left(X^{f}(s)\right) d s=n\right\} \quad \text { for } \quad n \in \mathbb{N} .
$$

Then, we choose an arbitrary strategy $\boldsymbol{f} \in \mathcal{A}(x)$, and from Itô's formula we write by inserting $X^{f}(\cdot)$ into function $F$

$$
\begin{aligned}
F\left(X^{f}\left(\tau_{n}^{f}\right)\right)= & F(x)+\int_{0}^{\tau_{n}^{f}}\left\{\mathcal{L}^{f} F\left(X^{f}(s)\right)\right\} d s \\
& -\int_{0}^{\tau_{n}^{f}} X^{f}(s) \boldsymbol{f}\left(X^{f}(s)\right) \sigma_{1} F_{x}\left(X^{f}(s)\right) d W_{1}(s) \\
& +\int_{0}^{\tau_{n}^{f}} X^{f}(s)\left(1+\boldsymbol{f}\left(X^{f}(s)\right)\right) \sigma_{2} F_{x}\left(X^{f}(s)\right) d W_{2}(s) .
\end{aligned}
$$

When we take the conditional expectation of the above terms, the stochastic integrals vanish because we have

$$
\begin{aligned}
& \mathbb{E}_{x}\left[\int_{0}^{\tau_{n}^{f}}\left(X^{f}(s) \boldsymbol{f}\left(X^{f}(s)\right) \sigma_{1} F_{x}\left(X^{f}(s)\right)\right)^{2} d s\right] \\
\leq & \sigma_{1}^{2}\left(1+\gamma_{+}\right)^{2} \frac{U^{2 \gamma_{+}+1}}{\left(L^{1+\gamma_{+}}-U^{1+\gamma_{+}}\right)^{2}} \mathbb{E}_{x}\left[\int_{0}^{\tau_{n}^{f}} f^{2}\left(X^{f}(s)\right) d s\right] \\
\leq & \sigma_{1}^{2}\left(1+\gamma_{+}\right)^{2} \frac{U^{2 \gamma_{+}+1}}{\left(L^{1+\gamma_{+}}-U^{1+\gamma_{+}}\right)^{2}} n<\infty,
\end{aligned}
$$

and

$$
\begin{aligned}
& \mathbb{E}_{x}\left[\int_{0}^{\tau_{n}^{f}}\left(X^{f}(s) \sigma_{2} F_{x}\left(X^{f}(s)\right)\right)^{2} d s\right] \\
\leq & \sigma_{2}^{2}\left(1+\gamma_{+}\right)^{2} \frac{U^{2 \gamma_{+}+1}}{\left(L^{1+\gamma_{+}}-U^{1+\gamma_{+}}\right)^{2}}<\infty .
\end{aligned}
$$

Then, it follows from (B.10) and (B.11) that

$$
\mathbb{E}_{x}\left[\int_{0}^{\tau_{n}^{f}}\left(X^{f}(s)\left(1+\boldsymbol{f}\left(X^{f}(s)\right)\right) \sigma_{2} F_{x}\left(X^{f}(s)\right)\right)^{2} d s\right]<\infty,
$$


The function $F$ solves the HJB equation. Thus, we have, $\forall \boldsymbol{f} \in \mathbb{R}$ and for each $s \mathbb{P}$-a.s. the inequality

$$
\left\{\mathcal{L}^{f} F\left(X^{f}(s)\right)\right\} \leq 0 .
$$

By using the above inequality we obtain

$$
F(x) \geq \mathbb{E}_{x}\left[F\left(X^{f}\left(\tau_{n}^{f}\right)\right)\right] .
$$

Notice that $F$ is a probability function. That is, $F(x) \in[0,1]$ for $x \in[L, U]$. In addition, we have as $n \rightarrow \infty, \tau_{n}^{f} \rightarrow \tau^{*}$. Therefore, from the dominated convergence theorem, we have as $n \rightarrow \infty$

$$
\mathbb{E}_{x}\left[F\left(X^{f}\left(\tau_{n}^{f}\right)\right)\right] \rightarrow \mathbb{E}_{x}\left[F\left(X^{f}\left(\tau^{*}\right)\right)\right] .
$$

We can then write the inequality

$$
\begin{aligned}
F(x) & \geq \mathbb{E}_{x}\left[F\left(X^{f}\left(\tau^{*}\right)\right)\right] \\
& =\mathbb{P}_{x}\left(\tau_{U}^{f}<\tau_{L}^{f}\right),
\end{aligned}
$$

where we used the boundary conditions for the last line. When we take the supremum over admissible strategies, we have

$$
F(x) \geq \sup _{f \in \mathcal{A}(x)} \mathbb{P}_{x}\left(\tau_{U}^{f}<\tau_{L}^{f}\right)=F(x),
$$

showing us that $\boldsymbol{f}^{*}(x)$ is the optimal leverage strategy and $F(x)$ is the optimal value function. In fact, with $\boldsymbol{f}^{*}\left(X^{*}(s)\right)$, the supremum of the term $\left\{\mathcal{L}^{f} F\left(X^{f}(s)\right)\right\}$ is attained. The inequality in (B.15) becomes equality, proving the optimality of our results.

\section{Signs of $\beta$ and The Risk Aversion Parameters}

We provide the proof for the first case that corresponds to the case when $\theta<\boldsymbol{f}^{*}$. The extension to second case is straightforward.

In the proof of the second problem, we obtained the quadratic equation

$$
\gamma^{2} \sigma^{2}\left[\sigma^{2}(\alpha(\rho \kappa-1))^{2}-\sigma_{2}^{2}\right]-2 \gamma \sigma^{2}[(b-c)+(b-r) \alpha(\rho \kappa-1)]+(b-r)^{2}=0,
$$

and used the root $\gamma_{+}<0$ to find the optimal results. This is the root that corresponds to

$$
\frac{1}{2 a_{1}}\left[-a_{2}+\sqrt{a_{2}^{2}-4 a_{1} a_{3}}\right] .
$$

By using the specification above we can check that having $\gamma^{+}<-1$ is equivalent to having $a_{3}>a_{2}-a_{1}$. When the relevant values in the quadratic function is substituted to the aforementioned inequality, we obtain $\beta>0$. Furthermore, when $\gamma_{+}=-1$, we have $a_{3}=a_{2}-a_{1}$, giving $\beta=0$. Finally, when $-1<\gamma_{+}<0$, we have $a_{3}<a_{2}-a_{1}$, which implies $\beta<0$. 


\section{Monotonicity of $F(x)$ in $\gamma$}

For any $\gamma \in \mathbb{R} \backslash\{-1\}$, we have the probability function $F(x)$ given by

$$
F(x)=\frac{L^{1+\gamma}-x^{1+\gamma}}{L^{1+\gamma}-U^{1+\gamma}} \quad \text { for } x \in[L, U] .
$$

Pick any $\gamma^{\prime} \leq \gamma^{\prime \prime}$. Then, $1+\gamma^{\prime} \leq 1+\gamma^{\prime \prime}$. It follows that,

$$
\left(1+\gamma^{\prime}\right) \ln \left(\frac{x}{L}\right) \leq\left(1+\gamma^{\prime \prime}\right) \ln \left(\frac{x}{L}\right) \quad \text { for } L<x
$$

From above, we can write $(x / L)^{1+\gamma^{\prime}} \leq(x / L)^{1+\gamma^{\prime \prime}}$ and

$$
1-\left(\frac{x}{L}\right)^{1+\gamma^{\prime}} \geq 1-\left(\frac{x}{L}\right)^{1+\gamma^{\prime \prime}} .
$$

Notice that the specification in equation (D.1) can be written as

$$
F(x)=\frac{1-\left(\frac{x}{L}\right)^{1+\gamma}}{1-\left(\frac{U}{L}\right)^{1+\gamma}} .
$$

By using (D.2) we observe that both the numerator and the denominator is monotonically decreasing in $\gamma$. When we check the limits, we obtain $\lim _{\gamma \downarrow-\infty} F(x)=1$ and $\lim _{\gamma \uparrow \infty} F(x)=$ 0 . We apply L'Hôpital's rule to find the latter result. This gives

$$
\lim _{\gamma \uparrow \infty}\left(\frac{x}{U}\right)^{1+\gamma} \frac{\ln (x)-\ln (L)}{\ln (U)-\ln (L)}=0,
$$

showing that the numerator declines faster than the denominator for increasing $\gamma$. Therefore, we have for $\gamma^{\prime} \leq \gamma^{\prime \prime}$

$$
\frac{1-\left(\frac{x}{L}\right)^{1+\gamma^{\prime}}}{1-\left(\frac{U}{L}\right)^{1+\gamma^{\prime}}} \geq \frac{1-\left(\frac{x}{L}\right)^{1+\gamma^{\prime \prime}}}{1-\left(\frac{U}{L}\right)^{1+\gamma^{\prime \prime}}},
$$

proving that $F(x)$ is a monotonically decreasing function in increasing $\gamma$.

\section{References}

Björk, T., 2004. Arbitrage Theory in Continous Time. Oxford University Press.

Browne, S., 1999. Beating a moving target: Optimal portfolio strategies for outperforming a stochastic benchmark. Finance and Stochastics 3, 275-294. 
Caner, M., Grennes, T., Koehler-Geib, F., 2010. Finding the tipping point when sovereign debt turns bad. Policy Research Working Paper Series 5391, The World Bank.

Cecchetti, S., Mohanty, M., Zampolli, F., 2011a. Challenges to central banking in the context of the financial crisis. Academic Foundation: New Delhi, Ch. The future of public debt, pp. 183-217.

Cecchetti, S., Stephen, G., Mohanty, M., Zampolli, F., 2011b. The real effects of debt. Working paper, Bank for International Settlements (BIS).

Feenstra, Robert C., R. I., Timmer, M. P., 2013. The next generation of the penn world table. Available for download at www.ggdc.net/pwt.

Fleming, W., Soner, H., 1993. Controlled markov processes and viscosity solutions. Springer, Berlin, New York.

Fleming, W. H., Stein, J. L., 2001. Stochastic inter-temporal optimization in discrete time. In: Takashi, N., Ramachandran, R., Mino, K. (Eds.), Economic Theory Dynamics and Markets: Essays in Honor of Ryuzo Sato. Kluwer, Boston.

Fleming, W. H., Stein, J. L., 2004. Stochastic optimal control, international finance and debt. Journal of Banking and Finance 28, 979-996.

Kourtellos, A., Stengos, T., Tan, C., 2013. The effect of public debt on growth in multiple regimes. Journal of Macroeconomics 38, Part A, 35-43.

Lane, P. R., Milesi-Ferretti, G. M., 2007. The external wealth of nations mark ii. Journal of International Economics 73 (2), 223-250.

Mankiw, N., Elmendorf, D., 1999. Government Debt. North Holland.

Merton, R. C., 1971. Optimum consumption and portfolio rules in a continuous-time model. Journal of Economic Theory 3, 373-413.

Merton, R. C., 1990. Continuous Time Finance. Blackwell, Massachusetts.

Reinhart, C. M., Rogoff, K. S., 2010. Growth in a time of debt. American Economic Review Papers and Proceedings 100 (2), 573-578.

Stein, J. L., 2005. Optimal debt and endogenous growth in models of international finance. Australian Economic Papers 44, 389-413.

Stein, J. L., 2006. Stochastic Optimal Control, International Finance and Debt Crises. Oxford University Press. 
Stein, J. L., 2007. United States current account deficits: A stochastic optimal control analysis. Journal of Banking and Finance 31 (5), 1321-1350.

Stein, J. L., 2010. A tale of two debt crises: A stochastic optimal control analysis. Economics: The Open-Access, Open-Assessment E-Journal 4.

Stein, J. L., 2011. The diversity of debt crisis in europe. Cato Journal 31 (2), 199-215.

Stein, J. L., 2012. Stochastic Optimal Control, and U.S. Debt Crises. Springer-Science.

Stein, J. L., Paladino, G., 2001. Country default risk: An empirical assessment. Australian Economic Papers 40 (4). 